\title{
Effects of inbreeding on morphological and life history traits of the sand cricket, Gryllus firmus
}

\author{
DEREK A. ROFF \\ Department of Biology, McGill University, 1205 Dr Penfield Ave., Montreal, Quebec, Canada, H3A $1 B 1$
}

\begin{abstract}
Inbreeding depression can cause substantial decreases in trait values. For colonizing organisms, which are likely to suffer relatively high levels of inbreeding at frequent intervals, inbreeding depression could significantly influence the evolution of traits and their genetic architecture. In the present paper, I examine inbreeding depression in the cricket Gryllus firmus, an inhabitant of ephemeral habitats such as sand dunes. Two questions are addressed: (i) do some traits show high levels of inbreeding depression; and (ii) do life history traits show higher levels of inbreeding depression than morphological traits? Growth rate and fecundity show very high levels of inbreeding depression ( $8-16 \%$ for the former, $14 \%$ for the latter). These rates of depression could potentially have significant effects on the survival probability of newly established populations. Overall, life history traits show significantly higher inbreeding depression $(5.3 \%)$ than morphological traits $(0.4 \%)$.
\end{abstract}

Keywords: additive variance, dominance variance, genetic architecture, inbreeding depression.

\section{Introduction}

The deleterious effects of inbreeding are widely recognized (Charlesworth \& Charlesworth, 1987; Thornhill, 1993). These effects have been most extensively investigated in plants (Husband \& Schemske, 1996), less so among vertebrates and, with the exception of Drosophila and Tribolium, very little among invertebrates (Roff, 1997).

Small population size and non-random mating will typically lead to inbreeding (for a review of the general effects of population bottlenecks on genetic architecture within populations, see Roff, 1997; and for analyses of the effects on variation among populations, see Wade \& McCauley, 1988; Whitlock \& McCauley, 1990; Pray \& Goodnight, 1995). The former phenomenon is particularly likely to occur in organisms that, because of environmental heterogeneity, must undergo frequent colonizing episodes. In this category can be placed wing dimorphic insects, which are characterized by a fully winged, volant morph and a flightless morph. A comparative analysis of wing dimorphic insects has shown them to be typically inhabitants of ephemeral habitats

Correspondence. E-mail: droff@bio1.lan.mcgill.ca such as early successional stages (Roff, 1990a; Denno et al., 1991). As a consequence of repeated colonization episodes, wing dimorphic insects are likely to pass through population bottlenecks frequently and, thus, be commonly subjected to inbreeding. The effects of inbreeding depression might, therefore, be of considerable consequence in the evolution of wing dimorphism.

Inbreeding depression occurs because of dominance: with dominance alone, the mean trait value declines linearly with the inbreeding coefficient (Falconer, 1989) but, when epistatic effects are also present, the decline can be non-linear (Crow \& Kimura, 1970). Morphological traits are generally determined primarily by additive genetic variance, whereas life history traits are frequently distinguished by large dominance/additive genetic variance ratios (Mousseau \& Roff, 1987; Crnokrak \& Roff, 1995; Roff, 1997). At first glance, this would seem to predict that life history traits will show higher levels of inbreeding depression than morphological traits, an observation made by Falconer (1989, p. 249) but not systematically tested. In fact, because the magnitude of inbreeding depression depends upon trait values rather than simply the 
dominance to additive genetic variance ratio, the aforementioned prediction does not immediately follow (see Discussion).

The cricket Gryllus firmus is a relatively large (approximately $0.7 \mathrm{~g}$ ) wing dimorphic cricket that inhabits sandy sites along the eastern seaboard of the United States (Harrison, 1985). Frequencies of the macropterous (long-winged, volant) morph range from $10 \%$ to $30 \%$ in the field (Veazy et al., 1976; Kevan, 1980), suggesting that this species frequently migrates to new habitats. In the present paper, I examine the effects of modest inbreeding (brother-sister) on both morphological and life history traits in G. firmus; first, to assess the effects of inbreeding in an invertebrate in which such effects could be evolutionarily important and, secondly, to test the hypothesis that morphological traits will show lower levels of inbreeding depression than life history traits.

\section{Materials and methods}

\section{Experimental protocol}

The individuals used in the present experiment were derived from a stock culture that originated from approximately 20 males and 20 females collected in northern Florida in 1981. The stock culture is maintained with a standing adult population of several hundred individuals (generally 100-500 adults, with occasional bottlenecks in which the population may have declined to about 50 adults). To prevent diapause, the temperature is maintained in excess of $25^{\circ} \mathrm{C}$. Nymphs and adults in both the stock and the experiment were fed Purina rabbit chow.

The grandparental generation for the present experiment was derived from eggs collected from dishes of earth placed in the stock cage. Eggs, nymphs and adults were raised throughout at $28^{\circ} \mathrm{C}$ and a photoperiod of $15 \mathrm{~h}$ light: $9 \mathrm{~h}$ dark. Nymphs were reared in batches of 40 in $4 \mathrm{~L}$ buckets $(20 \mathrm{~cm}$ diameter $\times 15 \mathrm{~cm}$ height). Upon eclosion to adults, male and female pairs were set up and, from these, full-sib families were obtained and reared as described above. These families were grouped into pairs and adults crossed as indicated in Fig. 1. Thus, from each group, two inbred families were formed by brother-sister mating, and two outbred families were formed by reciprocal matings of a male and a female from each family within the group. The advantage of this particular breeding design is that, within each group, there is an equal representation of alleles, only their combinations changing. Eggs
Family A

Family $B$
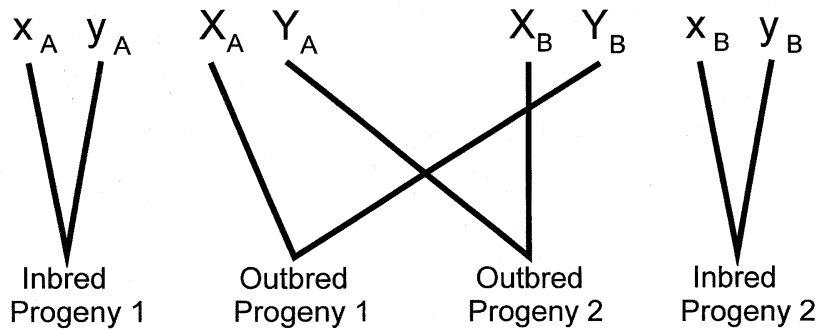

Fig. 1 Schematic illustration of the breeding design for a single 'group' ( $\mathrm{X}, \mathrm{x}$, female; $\mathrm{Y}, \mathrm{y}$, male; upper case indicates crosses between the two families, A and B, to produce outbred progeny, and lower case indicates brother-sister matings, producing inbred progeny).

and nymphs were raised as before, except that the 40 nymphs per family were split between two cages to permit removal of cage effects in the genetic analysis (see below). In total, 24 groups were used: of the 96 possible families, 71 were established, with 21 groups represented by at least one inbred and one outbred family (Table 1). The principal failure in the establishment of the families was caused by the female producing insufficient eggs or nymphs (18 cases). Other failures resulted from death of the male or female (three cases), escape of the female (one case) or no female parents available from the parental cage (three cases).

Table 1 Summary of group compositions used

\begin{tabular}{cccccc}
\hline Parental & $\begin{array}{c}\text { Nymphal } \\
\text { egg } \\
N_{\mathrm{I}}\end{array}$ & $N_{\mathrm{O}}$ & $\begin{array}{c}\text { traits } \\
\text { and adult } \\
\text { sample }\end{array}$ & $\begin{array}{c}\text { Fecundity } \\
\text { head width }\end{array}$ & $\begin{array}{c}\text { Offspring } \\
\text { egg } \\
\text { sample }\end{array}$ \\
\hline 2 & 2 & 12 & 8 & 8 & 4 \\
2 & 1 & 3 & 3 & 3 & 5 \\
1 & 2 & 7 & 6 & 5 & 8 \\
0 & 2 & 2 & 0 & 1 & 0 \\
2 & 0 & 0 & 1 & 1 & 1 \\
1 & 1 & 2 & 4 & 3 & 3 \\
0 & 1 & 0 & 2 & 3 & 2 \\
1 & 0 & 0 & 0 & 0 & 1 \\
0 & 0 & 0 & 0 & 0 & 0 \\
\hline
\end{tabular}

$N_{\mathrm{I}}$, number of families in a group formed by brothersister mating.

$N_{\mathrm{O}}$, number of families in a group formed by mating across the families. 
The following traits were measured.

1 Length of the egg on the first day it was laid ('parental egg sample'). From each female parent, 20 eggs were obtained and plated onto moist filter paper in a Petri dish. Eighty-one females produced at least 20 eggs (Table 1). Egg size at oviposition is strictly a female trait (Weigensberg et al., 1998) and, hence, for analysis, the mean egg length per female was used.

2 Length of the egg on the 10th day after oviposition. Egg size increases in length during development of the embryo and, by Day 10, there is a significant genetic contribution of the embryo to egg length (Weigensberg et al., 1998). However, because the length is still governed primarily by the maternal effect of initial size, the mean length per female was used as the basic datum.

3 Egg development rate. Development time from oviposition to hatching was recorded ( \pm 1 day) for the above eggs. Inbreeding depression, if it occurs, should increase development time. Because, in the analysis of inbreeding depression, the outbred value must be greater than the inbred value, the reciprocal of development time was used, which is the development rate (analysis using development time did not change any conclusions). As above, the mean value per female was used.

4 Egg survival: the proportion of the 20 eggs per female that hatched.

5 Nymphal head width at hatching. Nymphs were preserved at hatching, and head widths were measured later using a digitizer. As with egg size, the mean value per female was used, because the contribution of the maternal effect exceeds that of the contribution of the embryo (Weigensberg et al., 1998).

All of the following measurements were made on the individuals set up as described above.

6 Weights on Days 14, 21 and 28 after hatching. All nymphs were weighed individually at these three ages. The final age represents the midpoint of development, the mean time from hatching to final moult being approximately 50 days.

7 Survival to Days 14, 21, 28 and to final eclosion from hatching.

8 Development rate. As with egg development, the reciprocal of development time, development rate, was used to ensure that the predicted inbred value was less than the outbred value. The development time was also analysed and no differences were found with respect to the statistical significance of the difference between inbred and outbred values or the heritabilities. Cages were monitored every day except at the weekend: individuals moulting at the weekend were assigned to Sunday.

9 Adult head width. Adults were preserved after the final moult, and head width was measured using a digitizer.

10 Fecundity. First-week fecundity was assessed by ovary weight of virgin females. When kept as virgins, females of $G$. firmus produce but do not lay their eggs. Egg number is proportional to gonad weight (Roff, 1994), and the number of eggs produced by a virgin in the first 7 days after the final moult is not significantly different from the total egg production (eggs laid + eggs in ovaries) of a mated female (D. A. Roff, unpublished data). Attempts were made to obtain a minimum sample of five females per family, evenly distributed between replicate cages. The mean sample size was 5.72 females per family, distributed over 69 families (Table 1).

11 Because egg and hatching size are primarily maternal traits, these were measured from a sample of the offspring females ('offspring egg sample'). Samples were obtained from 65 families (Table 1), with an average of 1.9 females per family.

The above traits were classified into two groups, 'morphological traits' and 'life history traits', with the latter defined as traits, such as fecundity, viability, survival and development rate, that are invariably and directly connected with fitness (Roff \& Mousseau, 1987). Because weight at a particular age is a measure of development rate or growth trajectory, this was classified as a life history trait. Of the above traits, egg length (Days 1 and 10), nymphal head width at hatching and adult head width were classified as morphological traits and all others as life history traits.

The coefficient of inbreeding depression, $\delta$, is defined as,

$\delta=1-\frac{X_{\mathrm{O}}}{X_{\mathrm{I}}}$,

where $X_{\mathrm{O}}$ is the mean outbred value and $X_{\mathrm{I}}$ is the mean inbred value. $\delta$ was calculated in two ways: first, using each individual datum (i.e. individuals classified simply as 'inbred' or 'outbred' and ignoring group membership) and, secondly, using the inbred and outbred means for each group. For survival proportion per cage survival rates were used for the 'individual' analysis. Cage effects, if present, will inflate the variances of the groups but should not affect the estimates themselves. Because the null hypothesis is $\delta>0$, all tests were one-tailed. For the first method of estimation, both $t$-tests and Mann- 
Whitney tests were used and, for the second method, a paired $t$-test. Correction for multiple tests was performed using the sequential Bonferroni procedure.

In the present analysis, the focus is not specifically on individual results but on the pattern of variation: specifically, do life history traits show overall higher levels of inbreeding depression than morphological traits? To assess this, two statistical tests were used. First, the two groups were compared using a two-sample test (specifically the Mann-Whitney test, because, as will be shown, the variances were significantly different) and, secondly, a runs test. The latter test is a very conservative test and simply asks if the ranking of the two trait types is different from random.

\section{Heritability estimation}

For the purpose of estimating heritability, the data set was divided into that consisting of matings between full-sibs and that between separate families. In the first case, heritability was estimated using a one-way nested ANOVA with family effects tested using Satterthwaite's formula (Sokal \& Rohlf, 1995). Because of a reasonable balance in the data, there were no differences in the probabilities estimated using either the uncorrected or corrected $F$-statistic for the family effect. The standard errors of the heritabilities were estimated using the formula given in Becker (1992). In some cases, the ANOvA gave a significant family effect, but the estimated standard errors produced a confidence region on $h^{2}$ that included zero. Because the standard error estimates are only approximate (Becker, 1992), it is likely that, in these cases, they are overestimated. To provide a further check on the statistical significance of the $h^{2}$, the following randomization test was performed. Cages were assigned at random to a family and $h^{2}$ estimated from a nested ANOVA, the whole procedure being repeated 4999 times. The probability of obtaining the observed heritability by chance alone is estimated as $(N+1) / 5000$, where $N$ is the number of times $h^{2}$ from the randomized data set equals or exceeds the observed value (Manly, 1997; the additional 1 is to account for the observed value). In the absence of cage effects, the foregoing randomization test was carried out by randomizing individuals among families. Because the male and female in each parental pair are brother and sister, mating is assortative, which will potentially lead to inflated estimates of $h^{2}$. Other sources of upward bias in the full-sib estimate of $h^{2}$ are maternal effects and dominance variance, $V_{\mathrm{D}}$. In the absence of non-additive genetic effects (including maternal), the estimated additive genetic variance, $V_{\mathrm{A}}$, will be proportionally increased by $1+m$, where $m$ is the correlation between the breeding values of the mates (Falconer, 1989): for full-sibs, $m=0.5$. In the presence of dominance, the covariance between fullsibs is $1 / 2 V_{\mathrm{A}}(1+m)+1 / 4 V_{\mathrm{D}}$ (Crow \& Kimura, 1970). Because many of the traits examined here are predicted to include dominance variance and possibly maternal effects, correction for assortative mating is likely to be unreliable.

Within each group, the offspring of the two outbred families are double second cousins. Heritabilities can, in principle, be estimated using a nested ANOVA with 'family' nested within 'group' (and 'cage' nested within 'family'). Possibly because of the relatively low number of groups, estimates obtained using this approach were unreliable. Therefore, the following method was adopted: the outbred families were divided into two unrelated samples by arbitrarily assigning each family within a group to separate samples, and the heritability was estimated for each sample using one-way nested ANOVA. This method has the advantage that the standard error of the heritability can be estimated directly as the standard deviation of the two estimates [i.e. $\mathrm{SE}=\sqrt{ } \Sigma\left(h_{i}^{2}-h^{2}\right)^{2}$, where $h_{i}^{2}$ is the $i$ th estimate $(i=1,2)$ and $h^{2}$ is the mean of the two estimates].

Analysis of variance and, hence, also the estimation of heritability assumes that the character is distributed normally. Unless the data are highly skewed, non-normality is not a serious problem because, by the central limit theorem, the means will tend to normality (Sokal \& Rohlf, 1995).

Non-additive genetic effects can arise because of scale effects, most particularly if the trait values are log-normally distributed as a result of multiplicative interactions between loci (Falconer, 1989). Transformation of scale can remove apparent inbreeding depression (for example, see fig. 8.17 in Roff, 1997). In such cases, a logarithmic transformation removes apparent dominance variance and, hence, can change the estimate of heritability. Traits that show particularly large inbreeding depression values require careful scrutiny for possible scale effects. A general procedure for finding the best transformation is the Box-Cox method, which seeks a transformation within the family of power transformations (Sokal \& Rohlf, 1995):

$$
\begin{aligned}
& X^{\prime}=\left(X^{\lambda}-1\right) / \lambda \quad(\text { for } \lambda \neq 0) ; \\
& X^{\prime}=\ln X \quad(\text { for } \lambda=0) .
\end{aligned}
$$


Confidence limits for $\lambda$ can be found by maximum likelihood. A value of $\lambda=1$ indicates a linear transformation (i.e. no transformation is required).

\section{Results}

Inbreeding depression estimates: overview of the data

Adult head width was significantly different between the sexes, and development rate varied significantly according to wing morph and sex: in these cases, $\delta$ was computed for the separate categories, and a combined estimate weighting the individual estimates by sample size was also computed. For these cases, overall differences between inbred and outbred values were tested using ANOvA with sex and wing morph as categorical variables. Fecundity also varied significantly between wing morphs, but because of the relatively low sample size $\delta$ was estimated using the total sample with the raw fecundities or statistically corrected to a common mean. There is little difference between estimates: all the data are presented but only the combined head width and the adjusted fecundity are used in the statistical analysis.

Estimates of the inbreeding depression coefficient ( $\times 100$ for convenience) vary from -4.09 (survival of eggs laid by the parental generation) to 16.08 (weight on Day 28, Table 2). There is little difference between the estimates using the individual and

Table 2 Inbreeding depression values in the cricket Gryllus firmus

\begin{tabular}{|c|c|c|c|c|c|c|}
\hline \multirow[b]{2}{*}{ Trait* } & \multicolumn{2}{|c|}{$\delta \times 100$} & \multicolumn{2}{|c|}{ Sample size $\dagger$} & \multicolumn{2}{|c|}{$P$} \\
\hline & All & Paired & Inbred & Outbred & All & Paired \\
\hline Egg length (parental), Day 1 & -0.53 & -0.76 & 38 & 44 & $\mathrm{R}^{\S}$ & $\mathrm{R}$ \\
\hline Egg length (parental), Day 10 & 0.32 & 0.41 & 36 & 41 & 0.339 & 0.224 \\
\hline Egg development rate (parental)§ & 1.23 & 1.58 & 36 & 41 & 0.1 & 0.041 \\
\hline Egg survival (parental) & -4.09 & -4.96 & 38 & 44 & $\mathrm{R}$ & $\mathrm{R}$ \\
\hline Nymphal head width (parental) & 0.86 & 1.12 & 42 & 39 & 0.088 & 0.046 \\
\hline Weight on Day 14 & 10.84 & 10.87 & 1110 & 1207 & $<10^{-5}$ & 0.022 \\
\hline Weight on Day 21 & 7.64 & 8.96 & 971 & 1098 & $<10^{-5}$ & 0.021 \\
\hline Weight on Day 28 & 16.08 & 18.63 & 929 & 1044 & $<10^{-5}$ & $<10^{-4}$ \\
\hline Adult head width, female & 1.15 & 1.5 & 384 & 432 & 0.001 & 0.041 \\
\hline Adult head width, male & 0.89 & 0.91 & 399 & 479 & 0.024 & 0.156 \\
\hline Adult head width, combined & 1.02 & 1.19 & 783 & 911 & 0.0004 & 0.019 \\
\hline Development rate, $\mathbf{S W} \uparrow$ & 2.51 & 3.59 & 314 & 298 & 0.003 & 0.035 \\
\hline 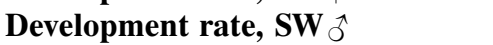 & 2 & 2.3 & 358 & 379 & 0.003 & 0.04 \\
\hline Development rate, $\mathrm{LW}+$ & 0.2 & 3.02 & 91 & 153 & 0.43 & 0.035 \\
\hline Development rate, $\mathbf{L W} \hat{\widehat{o}}$ & 3.03 & 5.24 & 54 & 104 & 0.021 & 0.01 \\
\hline Development rate & 2.02 & 3.12 & 817 & 934 & $<10^{-5}$ & 0.01 \\
\hline Survival to Day 14 & -0.08 & -1.11 & 68 & 74 & $\mathrm{R}$ & $\mathrm{R}$ \\
\hline Survival to Day 21 & 3.77 & 3.1 & 68 & 74 & 0.22 & 0.322 \\
\hline Survival to Day 28 & 3.16 & 3.01 & 68 & 74 & 0.28 & 0.347 \\
\hline Survival to adult & 4.81 & 5.84 & 68 & 74 & 0.15 & 0.43 \\
\hline Fecundity & 12.99 & 14.43 & 183 & 212 & 0.002 & 0.01 \\
\hline Adjusted fecundity & 14.2 & 13.03 & 183 & 212 & $<10^{-5}$ & 0.001 \\
\hline Egg length, Day 1 (offspring) & 0.49 & 0.47 & 58 & 64 & 0.258 & 0.323 \\
\hline Egg length, Day 10 (offspring) & 0.51 & 0.29 & 45 & 50 & 0.277 & 0.382 \\
\hline Egg development rate (offspring) & 2.53 & 2.15 & 45 & 50 & 0.043 & 0.088 \\
\hline Egg survival (offspring) & 5.25 & 4.06 & 45 & 50 & 0.125 & 0.243 \\
\hline Nymphal head width (offspring) & 0.41 & -0.01 & 44 & 45 & 0.261 & $\mathrm{R}$ \\
\hline
\end{tabular}

*Traits shown in bold are classed as 'life history traits'; those in regular type are 'morphological traits'. Because the hypothesis is that $\delta \geq 0$, all tests are one-tailed.

$\dagger$ Sample size for data sets using all individuals as data points. Sample sizes for paired comparisons vary between 19 and 23.

$¥$ All' refers to data set using all individuals as data points; 'paired’ refers to analysis using the mean value per group. $\S^{`} \mathrm{R}^{\prime}$ means that the hypothesis that $\delta \geq 0$ is rejected by virtue of a negative estimate. $P$-values shown are from $t$-tests or ANOva. The probabilities from the Mann-Whitney test did not differ. 


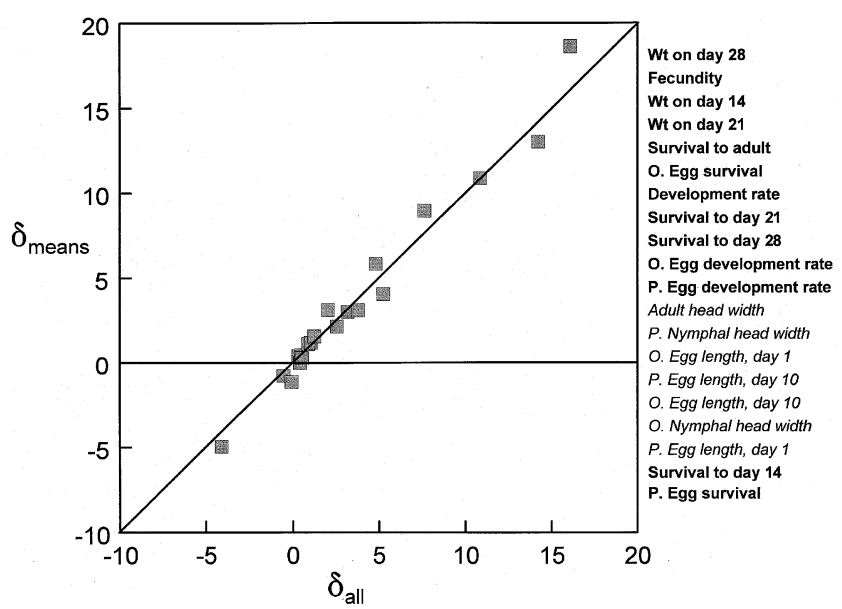

Fig. 2 A comparison of inbreeding depression coefficients for Gryllus firmus estimated using all data $\left(\delta_{\text {all }}\right)$ and values estimated using the group means $\left(\delta_{\text {means }}\right)$. The list on the right shows the ranking obtained using the group means. Life history traits are indicated by boldface type, morphological traits by italics.

the group means (Fig. 2), although the associated probability values of the former are smaller than the latter, as might be expected from the great difference in sample size (Table 2). After sequential Bonferroni correction, the only significant inbreeding depression values from the individual data set are weights on days 14, 21 and 28, development rate, fecundity and adult head width. The first five are life history traits and show higher levels of inbreeding depression than head width (10.8, 7.6, 16.1, 2.0, 13.6 and 1.0 respectively; Table 2). For the tests using group means, after correction for multiple tests, only the adjusted fecundity is significant.

\section{Inbreeding depression estimates: a comparison of morphological and life history traits}

The results did not differ between the data set based on all the observations from that based on the group means: only the latter analyses are presented here. Life history traits have a mean inbreeding depression value of $5.25 \%(n=13)$ compared with a value of $0.39 \%(n=7)$ for morphological traits. There is a highly significant difference in variances between the trait types $\left(F_{12,6}=87.8, P<0.0001\right)$ and, therefore, a difference in central tendency was tested for using the Mann-Whitney test, which showed a significant difference $\left(\chi_{1}^{2}=6.23, P=0.0126\right)$. The runs test supports the previous analysis: inbreeding depression values of the two types of traits are non-randomly distributed $(P<0.01$, Fig. 2$)$, with life history $\delta$ values being larger than morphological $\delta$ values.

\section{Heritability estimates}

None of the trait distributions was obviously highly skewed but, partly because of the large sample sizes typically involved, some of the distributions were statistically different from normal. The similarity between the parametric and non-parametric tests indicates that, in the present cases, the deviations from normality do not present a serious statistical problem.

The traits 'weight at age' and 'fecundity', which show particularly large inbreeding depression values, require careful scrutiny for possible scale effects. The appropriate transformation was found using the Box-Cox method (see Materials and methods). Estimates of $\lambda$ for weight at age (confidence limits in parentheses) of the outbred crickets are: age 14, $\lambda=0.74$ (0.62-0.86); age $21, \lambda=1.36$ (1.24-1.49); age $28, \lambda=0.89(0.78-1.01)$. The first two estimates of $\lambda$ are significantly different from 1 (no transformation) but also highly significantly different from zero (log transformation). For the outbred fecundity data, the estimates are: unadjusted fecundity, $\lambda=1.33$ (1.08-1.59); adjusted fecundity, $\lambda=1.01$ (0.72-1.32). In both cases, the estimates of $\lambda$ are very different from zero but close to unity. These results suggest that the large inbreeding depression values do not arise because of a scale effect. Heritability estimates obtained for the transformed and untransformed data are very similar and, following the dictum of Falconer (1989, pp. 297-298) that 'The first purpose of experimental observations is the description of the genetic properties of the population, and a scale transformation obscures rather than illuminates the description', the results for the untransformed data are presented.

Heritability estimates of growth rate (weight on day $t$ ), adult head width and development rate were all significantly different from zero for both the outbred and inbred data sets (Table 3). Heritabilities of survival approached significance (particularly as judged from the randomization or ANOVA tests), and the unadjusted fecundity is significant in the inbred data set (Table 3). As expected, the heritabilities from the outbred data set are lower than the unadjusted values from the inbred data set (Fig. 3). After adjustment using $m=0.5$, the 'inbred' heritability estimates are generally smaller than the 'outbred' heritability estimates (Fig. 3). The mean and median ratios of inbred $h^{2}$ to outbred $h^{2}$ are 0.86 and 0.89 , 
Table 3 Heritability estimates $(\times 100)$ of traits in Gryllus firmus

\begin{tabular}{|c|c|c|c|}
\hline Trait & 'Outbred' $h^{2}$ (SE) & 'Inbred' $h^{2}(\mathrm{SE})^{*}$ & $P_{\mathrm{R}} \dagger$ \\
\hline Weight on Day 14 & $45(8)$ & $40(13)$ & 0.001 \\
\hline Weight on Day 21 & $30(0.2)$ & $54(13)$ & $<10^{-4}$ \\
\hline Weight on Day 28 & $31(4)$ & $42(13)$ & 0.001 \\
\hline Adult head width, female & $48(25)$ & $54(15)$ & 0.004 \\
\hline Adult head width, male & $49(8)$ & 43 (11) & $<10^{-4}$ \\
\hline Development rate, $\mathrm{SW}$ + & $45(25)$ & $46(18)$ & 0.01 \\
\hline 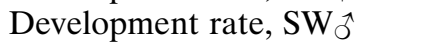 & $40(13)$ & $44(12)$ & $<10^{-4}$ \\
\hline Development rate, $\mathrm{LW}$ + & $22(19)$ & $54(23)$ & 0.001 \\
\hline Development rate, $\mathrm{LW} \widehat{o}$ & $48(20)$ & $45(35)$ & 0.08 \\
\hline Development rate, combined & $36(2)$ & $44(12)$ & $<10^{-4}$ \\
\hline Survival to Day 14 & $16(23)$ & $23(15)$ & 0.07 \\
\hline Survival to Day 21 & $25(25)$ & $31(17)$ & 0.04 \\
\hline Survival to Day 28 & $28(23)$ & $29(17)$ & 0.05 \\
\hline Survival to adult & $14(23)$ & $17(12)$ & 0.08 \\
\hline Unadjusted fecundity & $16(22)$ & $30(14)$ & 0.003 \\
\hline Adjusted fecundity & $17(23)$ & 12 (12) & 0.11 \\
\hline
\end{tabular}

*Estimates uncorrected for assortative mating.

$\dagger$ Probability from randomization test.

respectively, both substantially larger than the value of 0.67 predicted assuming no non-additive effects.

\section{Discussion}

Some of the heritabilities estimated for the traits examined in this study might be inflated by dominance and/or maternal effects. If they occur, the latter effects are most likely to be influential in traits found in early ontogeny (Riska et al., 1984; Montalvo \& Shaw, 1994). Egg size and size at hatching in G. firmus are largely maternally determined (Weigensberg et al., 1998), and weight at Day 14 might, therefore, be expected also to show maternal effects, these effects dissipating at later ages. There is, indeed, a decline in the heritability from 0.45 at Day 14 to 0.30 at Day 21 and 0.31 at Day 28. The similarity of the last two estimates suggests that maternal effects have largely dissipated by Day 21 . The large inbreeding depression values at all three ages indicate the presence of dominance variance: an estimate of weight at Day 28 from offspring on parent regression indicates that the full-sib estimate may be inflated by as much as 1.75 times (Table 4), giving a $V_{\mathrm{D}} / V_{\mathrm{A}}$ ratio of 1.5 . In contrast to weight at a particular age, survival to a particular age shows no clear pattern with age $(0.16,0.25,0.28$ and 0.14 for days 14, 21, 28 and to adult respectively), but the standard errors are so large (approximately 0.25) that equality of the values cannot be ruled out. Survival rates to Day 21 and beyond have a relatively large inbreeding depression (approximately 0.04 ), but none is significant (Table 2). Because of the large standard errors, more data are required to
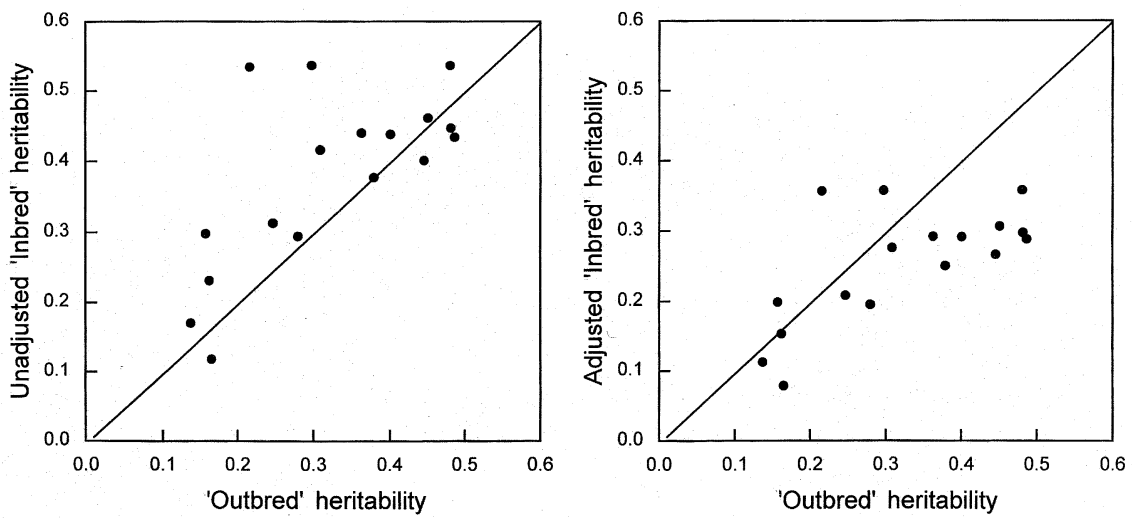

Fig. 3 A comparison for Gryllus firmus of the heritability estimates obtained using the outbred data with the estimates obtained using the inbred data. The plot on the left shows the heritabilities estimated from the inbred data before correction for assortative mating, the plot on the right the values after correction. 
determine adequately the inbreeding depression in, and genetic architecture of, survival.

Because of the experimental design, no estimates of the heritability of components before hatching were possible. Egg length at Day 1 or Day 10 and nymphal head width at hatching show no indication of inbreeding depression whether the mother is outbred and the embryos inbred (parental egg lengths) or if both mother and embryo are inbred (offspring egg lengths). This result is consistent with the prediction that morphological traits will have little dominance variance. In contrast to egg length and size at hatching, the inbreeding depression of egg development rate and survival of the nymph to hatching increase ( 0.01 to 0.03 and 0 to 0.05 respectively) when both the mother and the embryo are inbred (offspring eggs, Table 2). The lack of inbreeding depression when the mother is outbred and the embryo is inbred (parental eggs) argues for these traits being largely maternally determined.

Adult head width shows approximately the same inbreeding depression (approximately 0.01) as nymphal head width at hatching. The former has a full-sib heritability of 0.48 , which is typical of a morphological trait (Mousseau \& Roff, 1987). Offspring on parent and half-sib $h^{2}$ estimates of femur length, another morphological trait, indicate no detectable dominance variance (Table 4).

Development rate (hatching to adult) shows a low inbreeding depression (0.02), indicating little dominance variance. The full-sib heritability estimate from the present experiment (0.36) is the same as that for development time estimated by offspring on parent regression (0.36, Table 4), providing further evidence for the relative absence of dominance variance. On the other hand, fecundity, another trait closely connected to fitness, shows a substantial inbreeding depression (0.14) and a low full-sib heritability estimate $(0.16)$. Comparison of sire $(0.20)$ and dam (0.35) estimates from a half-sib experiment confirms the presence of non-additive effects (Table 4).

As expected, the heritability estimates from the inbred families generally exceed those from the

Table 4 A review of heritability estimates $(\times 100)$ for traits in Gryllus firmus (values presented in Table 3 not shown)

\begin{tabular}{llll}
\hline Trait & Estimation method* & $h^{2}(\mathrm{SE})$ & Reference \\
\hline Femur length & O-P & $45(18)$ & Unpublished data \\
& Offspring on sire & $41(25)$ & Unpublished data \\
& Offspring on dam & $50(23)$ & Unpublished data \\
& HS, sire estimate & $34(P=0.13) \dagger$ & Webb \& Roff (1992) \\
& HS, dam estimate & $33(P=0.04)$ & Webb \& Roff (1992) \\
& FS & $37(15)$ & Roff (1995a) \\
Head width & FS & $39(12)$ & Roff (1995a) \\
Weight at Day 28 & O-P & $16(15)$ & Unpublished data \\
& FS & $45(8)$ & Unpublished data \\
Weight at Day 35 & FS & $40(16)$ & Unpublished data \\
Development time & O-P & $>0$ & Roff (1990b) \\
& Offspring on dam & $36(8)$ & Roff (1995b) \\
& Offspring on sire & $57(11)$ & Roff (1995b) \\
& FS (morphs separate) & $34(11)$ & Roff (1995b) \\
& FS (morphs combined) & $32(1995)$ & Roff (1995b) \\
Fecundity & FS & $65(16)$ & Roff (1994) \\
& HS, sire estimate & $20(13) \ddagger$ & Roff et al. $(1997)$ \\
& HS, dam estimate & $35(13)$ & Roff et al. (1997) \\
\hline
\end{tabular}

\footnotetext{
*O-P, mean offspring on mid-parent; HS, half-sib; FS, full-sib; CR, correlated response to selection on development time.

$\dagger$ The estimates given in Webb \& Roff (1992) are in error, because they did not correct for cage effects. Because of the multiple nesting, standard errors were not calculated; instead, the probability values from the ANOVA are given. $\ddagger$ Both ANOva and randomization indicate a significant sire effect $(P=0.04)$, suggesting that the SE is overestimated (Roff et al., 1997).
} 
outbred families (Fig. 3). However, the degree of bias is less than expected based on assortative mating. On the basis of full-sib mating, the heritabilities from the inbred families should be approximately 1.5 times that estimated from the outbred families, whereas they are only 1.1 times as large. I can offer no explanation for this discrepancy.

The above results fit the observation made by Falconer (1989) that traits closely connected to fitness (life history) typically show higher levels of inbreeding depression than those less closely related to fitness (morphological). Falconer does not support this observation with a statistical analysis, although a detailed analysis does confirm it (M. A. DeRose and D. A. Roff, unpubl. data). A full discussion of the theoretical basis for this observation is beyond the scope of the present paper, but a simple example suffices to show that it is not an immediate consequence of dominance variance per se. Consider a trait determined by a single locus with two alleles. The inbreeding depression value is

$\delta=\frac{X_{\mathrm{O}}-\mathrm{X}_{\mathrm{I}}}{X_{\mathrm{O}}}=\frac{F \sqrt{V_{\mathrm{D}}}}{X_{\mathrm{O}}}$,

where $F$ is the inbreeding coefficient, $V_{\mathrm{D}}$ is the dominance variance, $X_{\mathrm{O}}$ is the mean outbred value and $X_{\mathrm{I}}$ is the mean inbred value. The inbreeding depression depends on the scaled dominance variance. Although there is evidence that the ratio of dominance to additive genetic variance increases in life history traits (Crnokrak \& Roff, 1995), the coefficient of dominance variance has not been examined. There is no simple algebraic relationship between $\delta$ and $V_{\mathrm{D}} / V_{\mathrm{A}}$, and numerical analysis shows that $\delta$ only increases with $V_{\mathrm{D}} / V_{\mathrm{A}}$ under particular conditions (D. A. Roff, unpubl. data).

Because it depresses the rate of population increase, the high inbreeding depression of growth rate, development rate and fecundity in G. firmus could have significant impacts on the survival probability of newly established populations. High levels of inbreeding depression on fitness-related traits have also been found in another colonizing species, Drosophila melanogaster (Mackay, 1985). Successive bouts of colonization should lead to a relative purging of deleterious recessives from the population, although as shown, even in the case of selffertilization, high levels of inbreeding depression can be preserved at mutation-selection balance (Lande \& Schemske, 1985; Charlesworth \& Charlesworth, 1987). More studies are required to determine if insects in general show high rates of inbreeding depression and if the rate in colonizing species is less than those from more stable habitats.

\section{Acknow ledgements}

I am most grateful to Martin Cayer without whose technical assistance this research would not have been possible. Funding for this work was provided by the Natural Science and Engineering Council of Canada.

\section{References}

BECKER, w. A. 1992. Manual of Quantitative Genetics. Academic Enterprises, Pulman, WA.

CHARLESWORTH, D. AND CHARLESWORTH, B. 1987. Inbreeding depression and its evolutionary consequences. Ann. Rev. Ecol. Syst., 18, 237-268.

CRNOKRAK, P. AND ROFF, D. A. 1995. Dominance variance: associations with selection and fitness. Heredity, 75, $530-540$.

CROW, J. F. AND KIMURA, M. 1970. An Introduction to Population Genetics Theory. Harper and Row, New York.

DENNO, R. F., RODERICK, G. K., OLMSTEAD, K. L. AND DOBEL, H. G. 1991. Density-related migration in planthoppers (Homoptera: Delphacidae): the role of habitat persistence. Am. Nat., 138, 1513-1541.

FALCONER, D. S. 1989. Introduction to Quantitative Genetics, 3rd edn. Longman, New York.

HARRISON, R. G. 1985. Barriers to gene exchange between closely related cricket species. II. Life cycle variation and temporal isolation. Evolution, 39, 244-259.

HUSBAND, B. C. AND SCHEMSKE, D. W. 1996. Evolution of the magnitude and timing of inbreeding depression in plants. Evolution, 50, 54-70.

KEVAN, D. M. 1980. The taxonomic status of the Bermuda beach cricket (Orthoptera: Gryllidae). Syst. Ent., 5, $83-95$.

LANDE, R. AND SCHEMSKE, D. W. 1985. The evolution of self-fertilization and inbreeding depression in plants. I. Genetic models. Evolution, 39, 24-40.

MANLY, B. F. J. 1997. Randomization, Bootstrap and Monte Carlo Methods in Biology. Chapman \& Hall, London.

MONTALVO, A. M. AND SHAW, R. G. 1994. Quantitative genetics of sequential life-history and juvenile traits in the partially selfing perennial Aquilegia caerulea. Evolution, 48, 828-841.

MOUSSEAU, T. A. AND ROFF, D. A. 1987. Natural selection and the heritability of fitness components. Heredity, 59, 181-198.

PRAY, L. A. AND GOODNIGHT, C. J. 1995. Genetic variation in inbreeding depression in the red flour beetle Tribolium castaneum. Evolution, 49, 176-188.

RiskA, B., ATChley, w. R. AND RUtLedGe, J. J. 1984. A genetic analysis of targeted growth in mice. Genetics, 107, 79-101.

ROFF, D. A. 1990a. The evolution of flightlessness in insects. Ecol. Monogr, 60, 389-421.

(c) The Genetical Society of Great Britain, Heredity, 81, 28-37. 
ROFF, D. A. 1990b. Understanding the evolution of insect life cycles: the role of genetical analysis. In: Gilbert, F. (ed.) Genetics, Evolution and Coordination of Insect Life Cycles, pp. 5-27. Springer-Verlag, New York.

ROFF, D. A. 1994. Evidence that the magnitude of the trade-off in a dichotomous trait is frequency dependent. Evolution, 48, 1650-1656.

ROFF, D. A. 1995a. The estimation of genetic correlations from phenotypic correlations: a test of Cheverud's conjecture. Heredity, 74, 481-490.

ROFF, D. A. 1995b. Antagonistic and reinforcing pleiotropy: a study of differences in development time in wing dimorphic insects. J. Evol. Biol., 8, 405-419.

ROFF, D. A. 1997. Evolutionary Quantitative Genetics. Chapman \& Hall, New York.

ROFF, D. A. AND MOUSSEAU, T. A. 1987. Quantitative genetics and fitness: lessons from Drosophila. Heredity, 58, 103-118.

ROFF, D. A., STIRling, G. AND FAIRBAiRn, D. J. 1997. The evolution of threshold traits: a quantitative genetic analysis of the physiological and life history correlates of wing dimorphism in the sand cricket. Evolution, 51, 1910-1919.
SOKAL, R. R. AND ROHLF, F. J. 1995. Biometry, 3rd edn. W. H. Freeman and Co., San Francisco.

THORNHILl, N. w. (ed.) 1993. The Natural History of Inbreeding and Outbreeding. University of Chicago Press, Chicago.

VEAZEY, J. N., KAY, C. A. R., WALKER, T. J. AND WHITCOMB, W. H. 1976. Seasonal abundance, sex ratio and macroptery of field crickets in northern Florida. Ann. Ent. Soc. Am., 69, 374-380.

WADE, M. J. AND McCAUley, D. E. 1988. Extinction and recolonization: their effects on the genetic differentiation of local populations. Evolution, 42, 995-1005.

WEBB, K. L. AND ROFF, D. A. 1992. The quantitative genetics of sound production in Gryllus firmus. Anim. Behav., 44, 823-832.

WEIGENSBERG, I., CARRIERE, Y. AND ROFF, D. A. 1998. Effects of male genetic contribution and paternal investment on egg and hatchling size in the cricket Gryllus firmus. J. Evol. Biol. (in press).

Whitlock, M. C. AND McCAuley, D. E. 1990. Some population genetic consequences of colony formation and extinction: genetic correlations within founding populations. Evolution, 44, 1717-1724. 